\title{
Original
}

\section{Manejo del SAHS mediante dispositivos de avance mandibular. Estudio preliminar}

\author{
Manuel Sánchez-Molinía,*, Angel Rollón ${ }^{a}$, José María Benítez ${ }^{b}$, Francisco Mayorga ${ }^{a}$, \\ Silvia Gallana ${ }^{a}$, Rodrigo Lozano ${ }^{a}$, Joel Joshi Otero ${ }^{a}$ y Magdalena Gascón ${ }^{c}$ \\ ${ }^{a}$ Cirugía Oral y Maxilofacial, Hospital Universitario Virgen Macarena, Sevilla, España. \\ bNeumología, Hospital Universitario Virgen Macarena, Sevilla, España. \\ 'Diplomada Universitaria en Enfermería, Hospital Universitario Virgen Macarena, Sevilla, España.
}

\section{INFORMACIÓN DEL ARTÍCULO}

\section{Historia del artículo:}

Recibido el 20 de octubre de 2010

Aceptado el 21 de diciembre de 2010

\section{Palabras clave:}

Dispositivo de avance mandibular;

Herbst;

Síndrome de apneas-hipopneas del sueño (SAHS);

Poligrafía

\begin{abstract}
R E S U M E N
El tratamiento que ha demostrado mayor eficacia en los pacientes con síndrome de apneas-hipopneas del sueño (SAHS) es la presión positiva continua de la vía aérea (CPAP). Los mayores inconvenientes son la incomodidad y la sensación de claustrofobia, que en algunos pacientes provoca rechazo o intolerancia. Una alternativa son los dispositivos de avance mandibular (DAM), que insertados en las arcadas dentarias producen el avance de la mandíbula y de la lengua, aumentando el volumen de la vía aérea.

Objetivo: Presentar nuestra experiencia en el tratamiento del SAHS mediante dispositivos de avance mandibular tipo Herbst.

Metodologia: Estudio de seguimiento prospectivo desde junio de 2006 hasta enero de 2009 de 7 pacientes del Área Hospitalaria Virgen Macarena con SAHS que rechazan el tratamiento con CPAP y a los que se ofrece tratamiento con DAM.

Las variables analizadas son: índice de apneas-hipopneas por hora, índice de desaturaciones por hora, intensidad subjetiva del ronquido y el test de somnolencia de Epworth, antes del tratamiento y al menos 6 meses después desde el inicio de su uso.

Utilizamos el test de Wilcoxon para detectar diferencias estadísticas significativas $(\mathrm{p}<0,05)$.

Resultados: Se observó una reducción estadísticamente significativa del índice de apneahipopneas por hora $(p<0,018)$ y del índice de desaturaciones por hora $(p<0,018)$, así como una reducción no significativa del ronquido y de la somnolencia.

Conclusiones: El uso de DAM tipo Herbst en pacientes afectos de SAHS que rechazan el uso de la CPAP podría ser útil, mejorando clínica y funcionalmente su situación.

(c) 2010 SECOM. Publicado por Elsevier España, S.L. Todos los derechos reservados.
\end{abstract}

\footnotetext{
${ }^{*}$ Autor para correspondencia.

Correo electrónico: manuelsmolini@gmail.com (M. Sánchez-Moliní González-Meneses).
} 


\title{
Sleep apnea-hypopnea syndrome management using a mandibular advancement device. Preliminary study
}

\author{
A B S T R A C T
}

Keywords:

Mandibular advancement device; Herbst,

Sleep apnea-hypopnea syndrome (SAHS);

Polysomnography
The most effective treatment in patients with sleep apnea-hypopnea syndrome (SAHS) is CPAP (continuous positive airway pressure). The main drawback of CPAP is the discomfort and claustrophobic sensation that it causes, which elicits rejection or intolerance by some patients. A non-surgical alternative to CPAP is the mandibular advancement device (MAD), which consists of a plastic splint inserted between the dental arches to shift the jaw and tongue forward and thus increase airway volume.

Objective: Report our experience with the treatment of SAHS using the Herbst mandibular advancement device.

Material and method: A prospective follow-up study was carried out from June 2006 until January 2009 at the Virgen Macarena University Hospital with 7 patients with SAHS who refused treatment with CPAP and were treated with the mandibular advancement device. The outcome variables analyzed were: apnea-hypopnea disruptions per hour index, desaturations per hour index, subjective intensity of snoring, and the Epworth Sleepiness Scale. Variables were evaluated pre-treatment and at least once 6 months after initiation MAD use. The Wilcoxon test for paired samples was used to detect statistically significant differences $(\mathrm{p}<0.05)$.

Results: A statistically significant reduction in the hourly indices of apnea-hypopnea disruptions $(p<0.018)$ and desaturations $(p<0.018)$ was observed, as well as a statistically nonsignificant reduction in snoring and sleepiness.

Conclusions: Use of the Herbst mandibular advancement device in patients with obstructive sleep apnea syndrome who refuse CPAP may be helpful as it improves the clinical and functional parameters of the condition.

() 2010 SECOM. Published by Elsevier España, S.L. All rights reserved.

\section{Introducción}

El síndrome de apnea obstructiva del sueño (SAOS) representa una entidad de gran trascendencia clínica y social caracterizada por la presencia de apneas (episodios de cese de paso de aire a través de la vía aérea superior) durante el sueño, como consecuencia de alteraciones anatómicas y funcionales que terminan provocando un colapso. Esto produce despertares transitorios, pérdida de la función fisiológica y reparadora del sueño, e hipersomnolencia diurna, siendo causa de cefaleas, pérdida de concentración, disfunciones sexuales, ronquidos, ahogos, hipertensión arterial, disfonía y otros tantos trastornos cardíacos, respiratorios y neuropsiquiátricos, además de ser una de las causas de accidentes de tráfico. La prevalencia de SAOS en España es del 4 al 6\% en hombres y del 2 al $4 \%$ en mujeres, y lo que es más importante, sólo están diagnosticados y tratados entre el 5 y el 9\% de los casos graves ${ }^{1}$.

El método de diagnóstico estándar es el estudio polisomnográfico donde, junto a numerosas variables clínicas (cardíacas, respiratorias y neurológicas), se detectan los episodios de apnea (ausencia o reducción $>90 \%$ de la señal respiratoria de $>10$ s de duración en presencia de esfuerzo respiratorio detectado por las bandas toracoabdominales) y de hipoapnea (reducción discernible entre el 30 y el 90\% de la amplitud de la señal respiratoria de $>10$ s de duración que se acompaña de una desaturación $\geq 3 \%$ y/o un microdespertar en el electroen- cefalograma) ${ }^{2}$, aunque cada vez se utiliza más la poligrafía ${ }^{1}$, que recoge únicamente variables cardiorrespiratorias.

En lugar de síndrome de apnea obstructiva es preferible utilizar la nomenclatura síndrome de apneas-hipopneas del sueño (SAHS), que se define como un cuadro de somnolencia excesiva, trastornos cognitivo-conductuales, respiratorios, cardíacos, metabólicos o inflamatorios secundarios a episodios repetidos de obstrucción de la vía respiratoria superior durante el sueño².

El SAHS se clasifica en leve si el índice de apneas/hipopneas por hora (IAHH) es de 5-15; moderado si el IAHH es de 15-30, y severo si el IAHH es $>30^{3}$. Los factores relacionados con la gravedad o severidad del SAHS son: edad, sexo (relación hombre: mujer, 3:1) en edades medias, obesidad, alcohol, tabaco, consumo de relajantes musculares y factores hormonales. Descartada la obstrucción anatómica evidente en la vía aérea (hipertrofia amigdalar o adenoidea, desviación de tabique nasal, hipertrofia de cornetes, etc.), el tratamiento que ha demostrado mayor eficacia es el uso de presión positiva continua de la vía aérea (CPAP) ${ }^{4}$, cuyo mayor inconveniente es la incomodidad y la sensación de claustrofobia, que provoca rechazo o falta de tolerancia en algunos pacientes. Otros tratamientos disponibles son las férulas de avance mandibular y los tratamientos quirúrgicos (turbinectomía, septoplastia, uvulopalatofaringoplastia, técnicas sobre el mentón, cirugía de hioides y cirugía ortognática) ${ }^{2}$.

Las férulas de avance mandibular son dispositivos de material plástico que, insertados en las arcadas dentarias, producen el avance de la mandíbula directamente e indirec- 


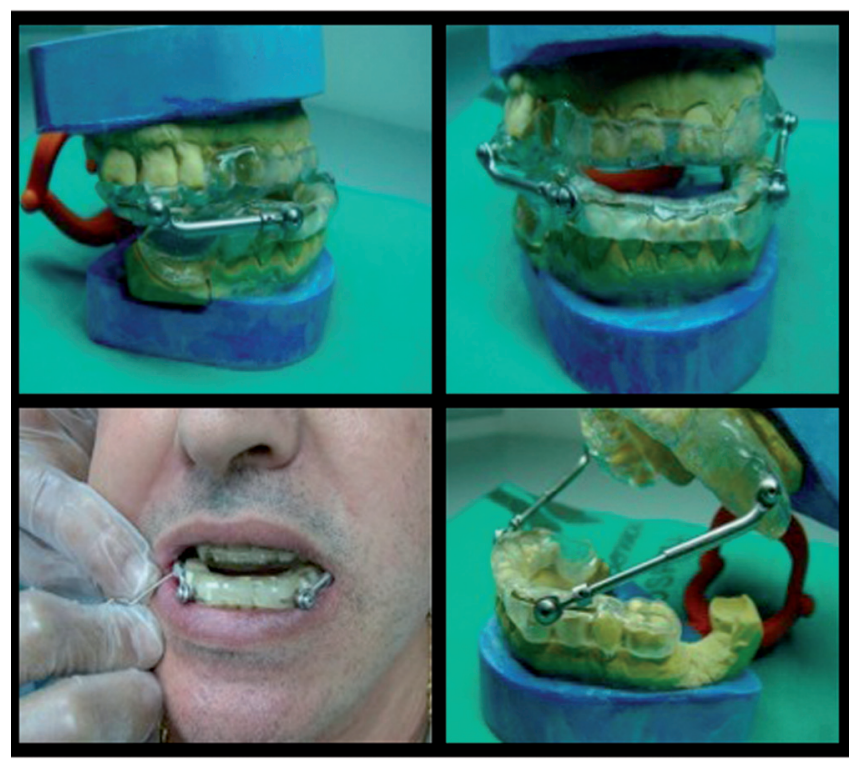

Figura 1 - Dispositivo de avance mandibular, antes de su colocación y produciendo el avance de la mandíbula en un paciente.

tamente sobre la lengua, el hioides y la musculatura suprahioidea, y por tanto el aumento de espacio y de la permeabilidad en la vía aérea superior (VAS) 5,6 (fig. 1).

Se considera que pueden ser una alternativa de tratamiento válida de primera elección para roncadores simples ${ }^{7}$, pacientes con SAHS leve ${ }^{4}$, pacientes con síndrome de resistencia aumentada de la vía respiratoria superior (SARVAS) ${ }^{8}$ y como segunda elección en pacientes que no responden 0 rechazan la $\mathrm{CPAP}^{4,9}$, pacientes con riesgo quirúrgico elevado y pacientes con escasa respuesta al tratamiento quirúrgico ${ }^{2}$.

\section{Objetivo}

Valorar la efectividad, la adherencia al tratamiento y las complicaciones con el uso de los dispositivos de avance mandibular tipo Herbst en el tratamiento del SAHS.

\section{Material y método}

Estudio de seguimiento prospectivo realizado por el Servicio de Cirugía Oral y Maxilofacial y Estomatología y la Unidad de Sueño del Servicio de Neumología del Área Hospitalaria Virgen Macarena de Sevilla, iniciado en junio de 2006 y que se prolongó hasta enero de 2009.

Los criterios de inclusión son pacientes diagnosticados por la Unidad del Sueño del Servicio de Neumología de SAHS mediante poligrafía respiratoria que tras el tratamiento inicial con presión positiva (CPAP) en revisiones posteriores no toleran o la rechazan, independientemente del grado de SAHS (leve, moderado o severo). Dichos pacientes son remitidos a la consulta de Cirugía Maxilofacial para valoración de tratamiento con dispositivos de avance mandibular.
El protocolo de estudio previo al tratamiento con férulas de avance mandibular incluye la recogida de las variables clínicas generales (edad, sexo, antecedentes patológicos personales, índice de masa corporal [IMC], hábitos tóxicos, hipertensión arterial), junto con la valoración del exceso de somnolencia diurna mediante la escala de somnolencia de Epworth (ESS) consistente en un test de 8 preguntas valoradas de 0-3 puntos cada una, cuya suma da un valor global que se considera normal si es menor a 12; la valoración subjetiva del ronquido medido a través de entrevista y respuesta de la pareja en una escala analógica subjetiva, medida de 0-10; y el estudio poligráfico que define el IAHH por hora de registro y el índice de desaturaciones por hora (IDH) de registro.

El protocolo de valoración por el Servicio de Cirugía Oral, Maxilofacial y Estomatología incluye estudio dentario (número de dientes presentes, dientes cariados, estado periodontal y tipo de oclusión), estudio de la articulación temporomandibular (presencia de crujidos, chasquidos, dolor a la apertura o a la masticación, bloqueos articulares, apertura oral máxima, protrusión máxima y lateralización máxima) y estudio radiológico que incluye ortopantomografía y telerradiografía lateral de cara. En caso de detectarse patología dentaria, el paciente es remitido para realizar tratamiento previo por su odontólogo habitual. Los criterios de exclusión de pacientes son: disponibilidad de menos de 2 dientes sanos en algunos de los sectores posteriores, enfermedad periodontal no tratada, movilidad dentaria grave (grado III) y presencia de signos o síntomas de patología de la articulación temporomandibular (ATM) (grado II-IV de Wilkes) ${ }^{10}$. Los pacientes con ruidos asintomáticos de la ATM (grado I de Wilkes), los pacientes con implantes dentales o prótesis fija, serán informados de los posibles riesgos por el efecto de la tracción del dispositivo y de la falta de evidencias de que se produzcan. Tras ser informado de posibles complicaciones asociadas a las férulas de avance y firmar el consentimiento, se le toman impresiones y mordidas en oclusión céntrica, máxima intercuspidación y máximo avance mandibular para la fabricación de la férula tipo Herbst, con vástago de avance regulable ${ }^{11}$. La férula tipo Herbst consta de dos fundas de acrílico individuales correspondientes a la arcada superior y a la arcada inferior, con planos oclusales planos que les permiten el deslizamiento de una sobre la otra. Dichas fundas son autoajustables y quedan encajadas y mantenidas en los dientes. Se mantienen unidas por unos vástagos metálicos con tope y con eje interno que le permiten incrementar la extensión progresivamente y cierta movilidad. Nuestro diseño dispone los vástagos con orientación de arriba abajo y de distal a mesial, de tal forma que el paciente encaja las fundas, se lo pone con la boca abierta y, una vez que cierra, el tope de los vástagos lleva la mandíbula hacia delante. Inicialmente fabricamos la férula con un avance del 50-60\% del máximo avance mandibular del paciente, lo que permite que vaya acomodándose al dispositivo. Si no manifiesta molestias, a las 2 semanas realizamos el avance completo mediante el desplazamiento de los vástagos. Si manifiesta molestias, vamos realizando un avance progresivo mensualmente hasta conseguir un desplazamiento máximo posible, siempre y cuando no presente ningún tipo de molestias, fundamentalmente en ATM. Una vez alcanzado el máximo de avance tolerable, se realiza el estudio 
poligráfico de control a los 3 meses postratamiento en el domicilio con el polígrafo cardiorrespiratorio Sibel Home Plus (Sibel Med), continuando con revisiones a los 6 meses y luego cada año ${ }^{12}$.

Las variables de resultados analizadas fueron: IAHH, IDH, somnolencia, medida por la escala de Epworth e intensidad del ronquido. Sus valores antes de tratamiento y a los 6 meses del uso del dispositivo de avance mandibular (DAM) fueron comparadas mediante el test de Wilcoxon para datos apareados, considerándose nivel de significación estadística un valor de $\mathrm{p}<0,05$. Se utilizó el programa SPSS versión 12.

\section{Resultados}

Desde junio de 2006 hasta enero de 2009, un total de 20 pacientes diagnosticados de SAHS procedentes de la Unidad de Sueño de nuestro hospital han sido remitidos a la Consulta de Cirugía Maxilofacial por intolerancia a la CPAP relacionada con claustrofobia o con molestias.

Diez (50\%) de estos 20 pacientes no cumplieron los criterios de inclusión para realizar tratamiento con férulas de avance mandibular debido a: mala salud bucodental (enfermedad periodontal avanzada con movilidad dental) en 3 pacientes, carencia de piezas dentales suficientes para insertar el dispositivo en 6 pacientes, o por rechazo explícito del tratamiento, 1 paciente.

Diez sujetos (50\%) fueron incluidos y se les fabricó el dispositivo de avance. De estos pacientes, 3 de ellos abandonaron la férula (33\%) antes de los 6 meses sin realizar la poligrafía postratamiento. Los motivos que propiciaron este abandono fueron sequedad de mucosa oral en 2 pacientes y molestias en la boca por el dispositivo en el otro paciente.

Los 7 pacientes restantes ( 5 hombres y 2 mujeres), con edad entre 36 y 67 años (media, 57; desviación estándar [DE], $9,3)$ la han usado a lo largo del periodo del estudio con estudios poligráficos pre y postratamiento. Cinco presentaban un SAHS grave y los otros 2 SAHS, moderado. El IMC previo al tratamiento osciló entre 26,2 y $45,91 \mathrm{~kg} / \mathrm{m}^{2}$, con media de 31,74 y DE de 7,08. El IMC postratamiento osciló entre 23,67 y $33,87 \mathrm{~kg} / \mathrm{m}^{2}$ (media, 29,33; $\mathrm{DE}, \pm 4,02$ ), sin diferencias significativas con el IMC pretratamiento. El seguimiento mínimo fue de 6 meses y el máximo de 31 meses (media, 13,28; DE, $\pm 10,14$ ). En el estudio poligráfico postratamiento (fig. 2) existe una reducción estadísticamente significativa $(p<0,05)$ del IAHH y del IDH (fig. 3). El IAHH mejora en todos los pacientes: 3 de los pacientes con SAHS severo pasan a leve y los 4 restantes mejoran sus índices, aunque se mantienen en su estatus pretratamiento ( 2 en grado severo y 2 en grado moderado) y presentan una reducción de la media del 51\%. En 4 pacientes se reduce más del $50 \%$ su IAHH (tabla 1). El índice IDH mejora en todos los pacientes y presenta una reducción relativa de la media del 52,3\%. La valoración subjetiva del ronquido y la somnolencia medida por la escala de Epworth mejoran de forma clínica sin llegar a la significación estadística (fig. 4). La intensidad del ronquido mejora de forma global en todos los pacientes, llegando a eliminarse por completo en 2 (mejoría en el 100\% de los casos y eliminación completa en el $28,6 \%$, con reducción relativa de la media del 63,8\%. La

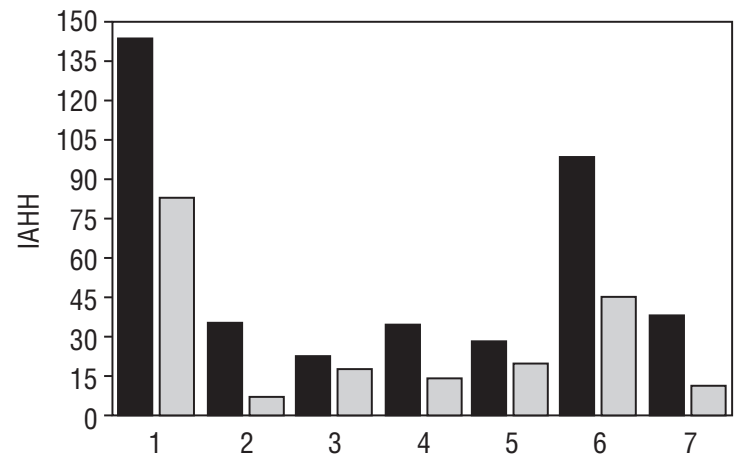

Figura 2 - Índice de apneas-hipopneas por hora (IAHH) en los pacientes del estudio: pretratamiento (columna oscura) y postratamiento (columna clara).

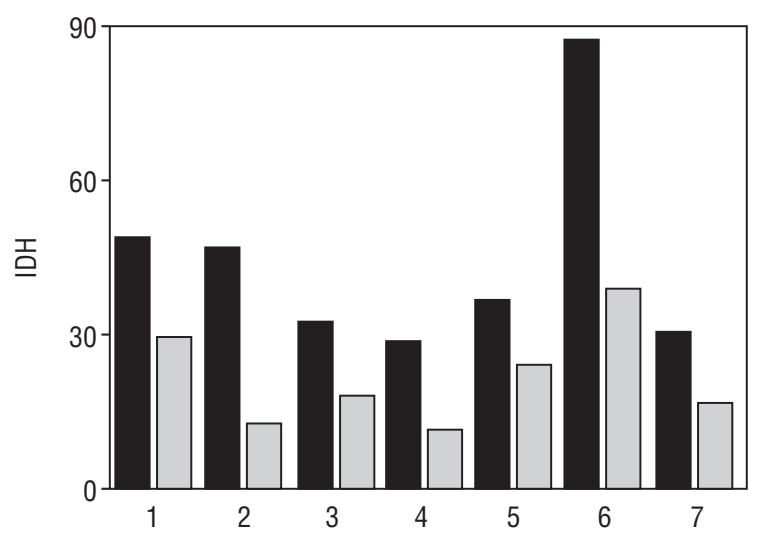

Figura 3 - Índice de desaturaciones por hora (IDH) en los pacientes del estudio: pretratamiento (columna oscura) y postratamiento (columna clara).

somnolencia mejora en 5 , no varía en 1 y empeora en 1 , con una mejora relativa de la media del 11,1\%.

En la valoración global de la muestra de pacientes que inician el tratamiento con DAM (10 pacientes), el 30\% de ellos se benefician del uso al cambiar de estatus.

No se analizaron factores de confusión.

\section{Discusión}

El mecanismo de acción de los DAM son el aumento del calibre y la disminución de la resistencia en la vía aérea gracias al avance de la mandíbula. Este movimiento de avance y rotación antihoraria (anteroinferior) provoca un efecto en la mandíbula fijándola, tensando y disminuyendo el grosor de la musculatura faríngea; de esta forma mantiene la rigidez y se reducen las vibraciones de los tejidos blandos ${ }^{13}$. Producen, asimismo, un aumento de la luz faríngea ${ }^{14}$. Existe un desplazamiento anterior también del paladar blando, de la lengua y del hueso hioides, evitando así su colapso ${ }^{9}$. La eficacia y los efectos colaterales dependen del tipo de dispositivo, del grado de avance y de la apertura bucal ${ }^{5}$. Algunos estudios consideran que el aparato de avance es mejor en una pieza ${ }^{15,16}$. 
Tabla 1 - Resultados pretratamiento y postratamiento: media (M), desviación estándar (DE), intervalo de confianza (IC) y significación estadística en test de Wilcoxon

\begin{tabular}{|c|c|c|c|c|}
\hline Variable & $\begin{array}{c}\text { Pretratamiento: } \\
\text { M (DE), (IC) }\end{array}$ & $\begin{array}{l}\text { Postratamiento: } \\
\text { M (DE), (IC) }\end{array}$ & Mejora relativa (M) & Test de Wilcoxon \\
\hline IMC & $\begin{array}{l}31,74(7,08) \\
(26,2-45,91)\end{array}$ & $\begin{array}{c}29,33(4,02) \\
(23,67-33,87)\end{array}$ & Reduce $7,6 \%$ & p: 0,06 \\
\hline IAHH & $\begin{array}{l}57,7(46,1) \\
(23-145)\end{array}$ & $\begin{array}{c}27,9(27,2) \\
(6,4-83)\end{array}$ & Reduce $51,7 \%$ & p: 0,018 \\
\hline IRH & $\begin{array}{c}61,8(71,1) \\
(0-183)\end{array}$ & $\begin{array}{l}22,4(30,2) \\
\quad(0-74)\end{array}$ & Reduce $73,8 \%$ & p: 0,08 \\
\hline IDH & $\begin{array}{c}44,8(20,5) \\
(29-88)\end{array}$ & $\begin{array}{c}21,4(10,1) \\
(11-39)\end{array}$ & Reduce $52,3 \%$ & p: 0,018 \\
\hline Epworth & $\begin{array}{c}11,7(3,4) \\
(7-16)\end{array}$ & $\begin{array}{c}9,7(5,3) \\
(0-16)\end{array}$ & Reduce $11,1 \%$ & p: 0,207 \\
\hline
\end{tabular}

IMC: índice de masa corporal; IAHH: índice de apneas-hipopneas por hora; IRH: índice de ronquidos por hora; IDH: índice de desaturaciones por hora.

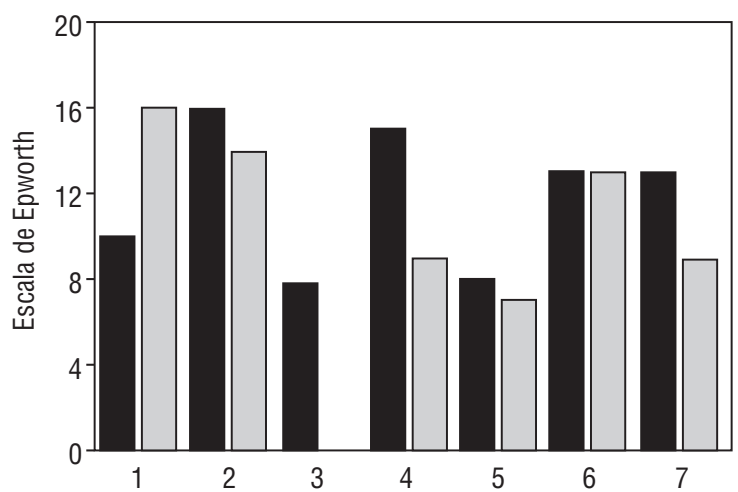

Figura 4 - Escala de Epworth en los pacientes del estudio: pretratamiento (columna oscura) y postratamiento (columna clara).

La férula tipo Herbst permite mantener la mandíbula en protrusión con la boca abierta y ciertos movimientos de lateralidad en comparación con otros dispositivos rígidos y de una sola pieza (tipo reposicionador mandibular). Son varios los trabajos que han demostrado su eficacia ${ }^{17,18}$ para reducir la severidad del SAHS. Su movilidad, al igual que el dispositivo Klearway ${ }^{11}$, permite a los pacientes realizar movimientos lentos y graduales adaptándolo a su cavidad oral, y en los pacientes que presentan disfunción temporomandibular resulta más confortable que los dispositivos que se insertan en una sola arcada dentaria. Asimismo los pacientes tienen menor sensación de claustrofobia y de restricción de la movilidad.

Los DAM también encuentran su indicación en el tratamiento del ronquido, desde su supresión en el $50 \%$ de los pacientes $^{19-21}$ hasta una reducción del $90-100 \%{ }^{21,22}$. Todos nuestros pacientes mejoraron la intensidad del ronquido, y en 2 de ellos (28,6\%) desapareció completamente.

Los efectos de los DAM sobre la somnolencia se han estudiado, aunque no de forma tan extensa como con la CPAP, por lo que se acepta que los dispositivos mejoran la somnolencia diurna aunque no necesariamente de manera superior a la CPAP ni a la uvulopalatofaringoplastia ${ }^{4,5,23}$. En nuestros pacientes se reduce en 5 , y la mejora relativa de la media es del $11,1 \%$.

La indicación primaria de los DAM son en SAHS levemoderado 4,9,10,24. En casos de SAHS leve-moderado con seguimiento de un año mejora significativamente el IAHH (el 78\% se normaliza y el $95 \%$ se reduce $\geq 50 \%)^{25}$. Aunque los SAHS graves pueden mejorar, su porcentaje de éxito es inferior ${ }^{20,21}$. En nuestra muestra de pacientes, 3 de ellos con SAHS grave mejoraron a SAHS leve, mientras que 2 de ellos $(28,6 \%)$ se mantuvieron en SAHS grave. Los otros 2 pacientes con SAHS moderado $(28,6 \%)$ se mantuvieron en su mismo grado. Aunque en el SAHS grave la variabilidad de los resultados con los DAM es alta, no encontramos razones para excluir a pacientes con SAHS grave ${ }^{26-28}$. El uso de estos dispositivos mejora el IAHH frente a la opción de no tratamiento ${ }^{4}$. En casos de SAHS leve-moderado con seguimiento de un año el IDH mejora significativamente ${ }^{25}$, al igual que en nuestra serie, donde mejora en todos los casos con significación estadística, a pesar de que en 5 casos el SAHS es grave.

Aunque la mayoría de guías-revisiones publicadas concluyen que la CPAP es más efectiva para controlar los trastornos respiratorios (IAHH y saturación de oxígeno) que los aparatos bucales $^{4,10}$, existen pacientes, como en nuestra serie, que no toleran la CPAP. Las indicaciones están establecidas en SAHS leve-moderado, en aquellos casos en los que no se tolera la $\mathrm{CPAP}^{4,10}$ y como factor predictivo de respuesta a cirugía ${ }^{29}$. En relación a los síntomas, a la calidad de vida y a la somnolencia no hay datos concluyentes ${ }^{4}$, aunque la férula parece tener mejores resultados en datos subjetivos y objetivos del sueño ${ }^{10}$, y son varios los trabajos donde los pacientes los prefieren a la $\mathrm{CPAP}^{4,5}$.

De los 10 pacientes que iniciaron el tratamiento con el dispositivo de avance mandibular (DAM), 3 (33\%) abandonaron el tratamiento debido a molestias ocasionadas por la sequedad de la mucosa oral y por molestias provocadas por el dispositivo. En la valoración global de los pacientes que iniciaron el estudio, a los que se les realizó el dispositivo, sólo el 
$30 \%$ se benefició de éste. No se produjeron abandonos por problema ATM, aunque durante el período de acomodación de la férula un paciente presentó molestias en la articulación que cedieron con tratamiento conservador: antiinflamatorios no esteroideos (AINE). El mayor inconveniente de los DAM es la molestia oral y, secundariamente, su abandono a causa de ésta. Izci et $\mathrm{al}^{23}$, en una revisión de pacientes con SAOS y roncadores tratados con férulas de reposición mandibular, refieren encontrar que los efectos colaterales y las molestias ocasionadas por su uso (dificultad para conciliar el sueño, molestias en la ATM, molestias en la boca, exceso de salivación, dificultad para la respiración y daño dental) ocurren en un $32-86 \%$ de los pacientes estudiados. Schmidt-Nowara et $\mathrm{al}^{19}$, en un su revisión sobre tratamiento con férulas de avance refieren que las molestias en la articulación temporomandibular son raras, mientras que la molestia oral es común, aunque disminuye con el uso y el ajuste de los dispositivos. Estos inconvenientes o complicaciones no se han podido relacionar (aumento de incidencia) con los protocolos de tratamiento más agresivos de avance ${ }^{26,30}$. La mayoría de los trabajos hablan de abandono de los DAM al año del 25-50\% de los pacientes ${ }^{5,25,31}$, aunque en alguno abandonan hasta el $88 \%{ }^{23}$.

El uso de la cirugía de la vía aérea superior genera controversias. Desde hace décadas se realizan diferentes técnicas quirúrgicas para aumentar el calibre de la vía aérea. Entre las distintas técnicas de cirugía de vía aérea superior se encuentran la traqueostomía, la uvulopalatofaringoplastia (UPPP) realizada o no junto con amigdalectomía, la UPPP asistida por láser, la ablación por radiofrecuencia (RFA), los implantes palatinos, el avance maxilomandibular (MMA) mediante osteotomías de maxilar y mandíbula y la cirugía multinivel en dos fases. Una reciente revisión sistemática publicada por la Academia Americana de Medicina del Sueño ${ }^{32}$ (AASM) concluye que tras cirugía MMA se detecta una reducción sustancial y consistente del IAHH. Los resultados obtenidos tras cirugía faríngea son menos consistentes ${ }^{32}$. Las nuevas técnicas de cirugía faríngeas y procedimientos multinivel parecen prometedores $^{32}$. En la guía de parámetros prácticos ${ }^{33}$, la AASM, siguiendo el sistema GRADE, recomienda como opción el tratamiento mediante la ablación por radiofrecuencia o los implantes palatinos en casos de SAHS leve que no toleren el tratamiento con CPAP o con DAM, o en los que éstos sean inefectivos ${ }^{33}$. El MMA también lo considera una opción dentro de la terapéutica en pacientes con SAHS severo que no toleren tratamiento con CPAP y cuando los DAM sean inefectivos o no desados ${ }^{33}$. Considera que éstos son más apropiados en el SAHS moderado ${ }^{33}$. La AASM ${ }^{33}$ concluye que hay ausencia de datos rigurosos de evaluación del tratamiento quirúrgico de la vía aérea superior y se necesitan más estudios de investigación de calidad para determinar la eficacia y la población beneficiada.

Hoffstein ${ }^{5}$, en una revisión de dispositivos orales para el tratamiento de trastornos respiratorios durante el sueño, encuentra que entre el 40 y el 50\% de los pacientes abandonan el tratamiento por los efectos secundarios de los dispositivos, entre ellos: exceso de salivación, dolor muscular, molestias en ATM, cambios oclusales y sequedad de boca. También recalca que la mayoría de las férulas son bien toleradas y confortables cuando son confeccionadas por personal cualificado, y a los 30 meses el 55-70\% de pacientes las utilizan. Sin embargo, a pesar de todos estos inconvenientes, algunos estudios demuestran la preferencia de los pacientes por los DAM frente a la CPAP ${ }^{4,5}$.

\section{Conclusiones}

La utilización de dispositivos de avance mandibular tipo Herbst podría ser una opción válida de tratamiento en el SAHS, tanto en pacientes con SAHS grave, moderado o leve, pudiendo mejorar, de manera estadísticamente significativa, los índices de apneas-hipopneas por hora y el índice de desaturaciones por hora, lo que supondría una mejora funcional.

La limitación principal del presente estudio es el tamaño muestral pequeño, que hace necesario elaborar nuevos estudios prospectivos con un tamaño muestral mayor que permita establecer conclusiones firmes con respecto a lo sugerido en el presente trabajo.

\section{Conflicto de intereses}

Los autores expresan que no presentan conflictos de intereses al redactar el manuscrito. Las fuentes de financiación no han tenido participación en el diseño del estudio, en la colección de datos, en el análisis o en la interpretación de éstos, en la redacción del manuscrito o en la decisión de enviarlo para su publicación.

\section{B I B L I O G R A F Í A}

1. Duran-Cantolla J, Martínez-Null C. Perspectivas futuras en el diagnóstico del síndrome de apneas-hipopneas durante el sueño. Med Clin. 2005;125:692-4.

2. Consenso nacional sobre Síndrome de Apneas-Hipopneas del sueño (SAHS). Arch Bronconeumol. 2005;41 Supl 4:1-80.

3. Marín Trigo JM, Arán Corbella X, Barbé Illa F, Biurrum Unzué O, Fiz Fernández JA, Jiménez Gómez A, et al. Normativa sobre el diagnóstico y tratamiento del síndrome de apnea obstructiva durante el sueño (SAOS). Recomendaciones de la Sociedad Española de Neumologia y Cirugía Torácica (SEPAR). Barcelona: Doyma; 1993.

4. Lim J, Lasserson TJ, Fleetham J, Wright J. Aparatos bucales para la apnea obstructiva del sueño. Biblioteca Cochrane Plus. 2006. N. ${ }^{\circ} 2$.

5. Hoffstein V. Review of oral appliances for treatment of sleepdisordered breathing. Sleep Breath. 2007;11:1-22.

6. Battagel JM, Johal A, L'Estrange PR, Croft CB, Kotecha B. Changes in airway and hyoid position in response to mandibular protrusion in subjects with obstructive sleep apnoea (OSA). Eur J Orthod. 1999;21:363-76.

7. Loube DI, Andrada T, Shanmagum N, Singer MT. Successful treatment of upper airway resistance syndrome with an oral appliance. Sleep Breath. 1997;2:98-101.

8. Guilleminault C, Stoohs R, Clerk A, Cetel M, Maistros P. A cause of excessive daytime sleepiness. The upper airway resistance syndrome. Chest. 1993;104:781-7.

9. Ferguson KA, Ono T, Lowe AA, al-Majed S, Love LL, Fleetham JA. A short term controlled trial of an adjustable oral appliance 
for the treatment of mild to moderate obstructive sleep apnea. Thorax. 1997;52:362-8.

10. Fritsch KM, Iseli A, Russi EW, Bloch KE. Side effects of mandibular advancement devices for sleep apnea treatment. Am J Respir Crit Care Med. 2001;164:813-8.

11. Lowe AA. Principles of oral appliance therapy for the management of sleep disordered breathing. Oral Maxillofac Surg Clin North Am. 2002;14:305-17.

12. Kushida CA, Morgenthaler TI, Littner MR, Alessi CA, Bailey D, Coleman J Jr, et al; American Academy of Sleep. Practice parameters for the treatment of snoring and obstructive sleep apnea with oral appliances: an update for 2005. Sleep. 2006;29: 240-3.

13. Hoekema A, Stegenga B, de Bont LG. Efficacy and co-morbidity of oral appliances in the treatment of obstructive sleep apneahypopnea: a systematic review. Crit Rev Oral Biol Med. 2004;15:137-55.

14. Ryan CF, Love LL, Peat D, Fleetham JA, Lowe AA. Mandibular advancement oral appliance therapy for obstructive sleep apnea: effect on awake caliber of the velopharynx. Thorax. 1999;54:972-7.

15. Bloch K, Iseli A, Zhang JN, Xie X, Kaplan V, Stoeckli PW, et al. A randomized, controlled crossover trial of two oral appliances for sleep apnea treatment. Am J Respir Crit Care Med. 2000;162: 246-51.

16. Rose E, Staats R, Virchow C, Jonas IE. A comparative study of two mandibular advancement appliances for the treatment of obstructive sleep apnoea. Eur J Orthod. 2002;24:191-8.

17. Clark GT. Sleep apnea. OSA and dental appliances. CDA J. 1988;16:26-33.

18. Clark GT, Arand D, Chung E, Tong D. Effect of anterior mandibular positioning on obstructive sleep apnea. Am Rev Respir Dis. 1993;147:624-9.

19. Schmidt-Nowara W, Lowe A, Wiegand L, Cartwrigth R, PerezGuerra F, Menn S. Oral appliances for the treatment of snoring and obstructive sleep apnea: a review. Sleep. 1995;18:501-10.

20. Schmidt-Nowara WW, Meade TE, Hays MB. Treatment of snoring and obstructive sleep apnea with a dental orthosis. Chest. 1991;99:1378-85.

21. O'Sullivan RA, Hillman DR, Mateljan R, et al. Mandibular advancement splint: an appliance to treat snoring and obstructive sleep apnea. Am J Respir Crit Care Med. 1995; 151:194-8.

22. Clark GT, Blumenfeld I, Yoffe N, Peled E, Lavie P. A cross over study comparing the efficacy of continuous positive airway pressure with anterior mandibular positioning devices on patients with obstructive sleep apnea. Chest. 1996;109:144783.

23. Izci B, Mc Donald JP, Coleman EL, Mackay TW, Douglas NJ, Engleman HM. Clinical audit of subjects with snoring and sleep apnoea/hypopnoea syndrome fitted with mandibular repositioning splint. Respir Med. 2005;99:337-46.

24. Henke KG, Frantz DE, Kuna ST. An oral elastic advancement device for obstructive sleep apnea. Am J Respir Crit Care Med. 2000;161:420-5.

25. Tegelberg A, Wilhelmsson B, Walker-Engstrom ML, Ringqvist M, Andersson L, Krekmanov L, et al. Effects and adverse events of a dental appliance for treatment of obstructive sleep apnoea. Swed Dental J. 1999;23:117-26.

26. Gotsopoulos H, Chen C, Quian J, Cistulli PA. Oral appliance therapy improves symptoms in obstructive sleep apnea: a randomized controlled trial. Am J Respir Crit Care Med. 2002;166:743-8.

27. Walker-Engstrom ML, Ringqvist I, Vestling O, Wilhelmsson B, Tegelberg A. A prospective randomized study comparing two different degrees of mandibular advancement with a dental appliance in treatment of severe obstructive sleep apnea. Sleep Breath. 2003;7:119-30.

28. Fransson AM, Tegelberg A, Leissner L, Wenneberg B, Isacsson G. Effects of a mandibular protruding device on the sleep of patients with obstructive sleep apnea and snoring problems. A 2 year follow up. Sleep Breath. 2003;7:131-41.

29. Hoekema A, de Lange J, Stegenga B, de Bont LG. Oral appliances and maxillomandibular advancement surgery: an alternative treatment protocol for de obstructive sleep apnea-hypopnea syndrome. J Oral Maxillofac Surg. 2006;64:886-91.

30. Mehta A, Qian J, Petocz P, Darendeliler MA, Cistulli PA. A randomized, controlled study of a mandibular advancement splint for obstructive sleep apnea. Am J Respir Cri Care Med. 2001;163:1457-61.

31. Marklund $M$, Stenlund $H$, Franklin KA. Mandibular advancement devices in 630 men and women with obstructive sleep apnea and snoring. Tolerability and predictors of treatment success. Chest. 2004;125:1270-8.

32. Caples SM, Rowley JA, Prinsell JR, Pallanch JF, Elamin MB, Katz SG, et al. Surgical modifications of the upper airway for obstructive sleep apnea in adults: a systematic review and meta-analysis. Sleep. 2010;33:1396-407.

33. Aurora RN, Casey KR, Kristo D, Auerbach S, Bista SR, Chowdhuri $S$, et al. Practice parameters for the surgical modifications of the upper airway for obstructive sleep apnea in adults. Sleep. 2010;33:1408-13. 